\title{
Possible toxicity following embolization of congenital giant vertex hemangioma: case report
}

\author{
Ingrid Kieran, MD, ${ }^{1}$ Zaitun Zakaria, MD, ${ }^{2}$ Chandrasekaran Kaliaperumal, FRCSEd, ${ }^{2}$ \\ Declan O'Rourke, MRCP, ${ }^{3}$ Alan O'Hare, FRCR, ${ }^{4}$ Eoghan Laffan, FFR, ${ }^{5}$ John Caird, FRCSI(SN), ${ }^{2}$ \\ Mary D. King, FRCPI, FRCPCH, ${ }^{3}$ and Dylan J. Murray, FRCS, FDS, FFD ${ }^{1}$
}

\begin{abstract}
Departments of ${ }^{1}$ Plastic and Craniofacial Surgery, ${ }^{2}$ Neurosurgery, ${ }^{3}$ Pediatric Neurology, and ${ }^{5}$ Radiology, Temple Street Children's University Hospital; and ${ }^{4}$ Department of Neuroradiology, Beaumont Hospital, Dublin, Ireland
\end{abstract}

\begin{abstract}
The authors describe the case of a 3-year-old boy with a giant congenital vertex hemangioma who underwent presurgical embolization with Onyx (ethylene-vinyl alcohol copolymer dissolved in dimethyl sulfoxide) and Glubran ( $N$-butyl2-cyanoacrylate). This vascular tumor had no intracranial vascular communication as assessed by pre-embolization MRI and catheter angiography. All embolizations were performed by direct percutaneous injection. One week following the last embolization procedure the child presented with a 24-hour history of ataxia and extrapyramidal tremor. He was diagnosed with a possible immune-mediated reaction to Onyx or Glubran, which was treated with an urgent surgical excision of the hemangioma followed by intravenous administration of immunoglobulin and steroids. To the authors' knowledge, this is the first case of possible immune-mediated toxicity secondary to either Onyx or Glubran administration. This case highlights the need for awareness of potential toxic reactions to these embolic agents in the treatment of hemangiomas in the pediatric patient.
\end{abstract}

https://thejns.org/doi/abs/10.3171/2016.5.PEDS13345

KEY WORDS hemangioma; congenital; embolic agent; embolization; toxicity; vascular disorders

$\mathrm{N}$ ONINVOLUTING congenital hemangiomas (NICHs) are described as part of the spectrum of vascular tumors. ${ }^{3}$ They differ from rapidly involuting congenital hemangiomas, which proliferate rapidly in the 1st year of life and then gradually regress over the following 5 to 10 years. NICHs in contrast, fail to involute and histologically stain negative for glucose transporter protein 1 (GLUT-1). ${ }^{6}$ Hemangiomas are proliferative vascular lesions characterized by increased endothelial cell turnover, and NICHs therefore can result in large and highly vascularized lesions whereby surgical intervention poses considerable hemorrhage risk. In such cases, presurgical embolization can play a significant role., ${ }^{1,7}$ Pre-embolization work-up includes careful evaluation of the lesion using catheter angiography to assess for feeding and draining vessels. We present a case of a 3-year-old boy with a giant vertex hemangioma who developed systemic complica- tions following embolization with Onyx (ethylene vinyl alcohol copolymer) and Glubran ( $N$-butyl-2-cyanoacrylate).

\section{Case Report}

This 3-year-old boy was born with a small vertex scalp swelling. MRI performed shortly after birth showed that the scalp swelling was a highly vascular lesion that was completely extracranial. There was no particular abnormality of the brain or spine. The lesion grew steadily larger over the course of the 1st year and biopsy was performed when the patient was 1 year old. The biopsy showed fibroconnective tissue containing irregular, enlarged and dilated thin-walled vessels lined by plump endothelial cells. There was no evidence of atypia or malignancy. Immunohistochemical staining was negative for GLUT-1 and podoplanin, suggesting a NICH (Fig. 1).

Due to the gradual enlargement of the lesion and re- 

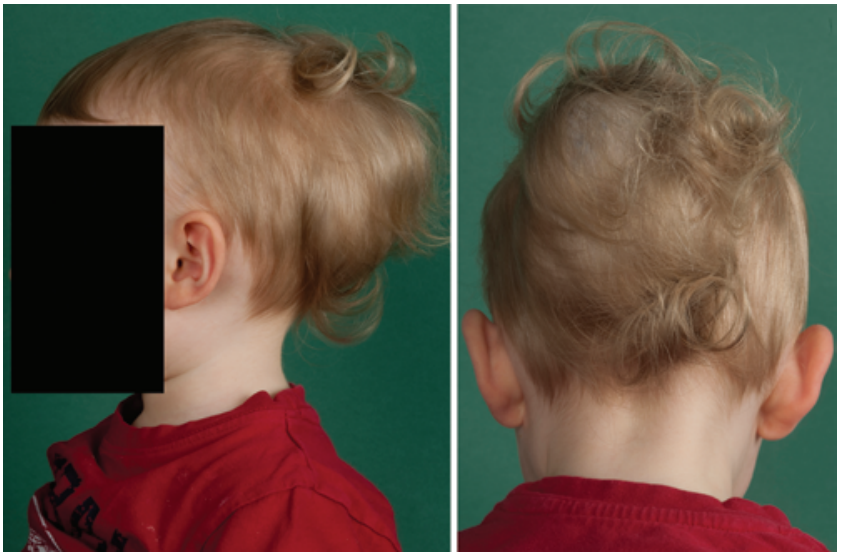

FIG. 1. Lateral (left) and posterior (right) photographs of a vertex hemangioma obtained when the patient was 18 months old. Figure is available in color online only.

sultant chronic hypochromic microcytic anemia, a trial of propranolol was commenced. Prior to propranolol treatment the lesion measured approximately $15 \times 15 \mathrm{~cm}$. There was no reduction in size after 6 months of treatment at a peak dose of $2.5 \mathrm{mg} / \mathrm{kg} / \mathrm{day}$, and elective resection of the hemangioma under the combined management of a neurosurgeon and craniofacial surgeon was therefore scheduled. This procedure was abandoned due to significant intraoperative hemorrhage, and subsequently a presurgical embolization was planned. Four-vessel cerebral angiography showed no evidence of cerebral vascular abnormality or vessels communicating with or feeding the scalp hemangioma (Fig. 2A). The patient subsequently underwent a course of percutaneous intralesional embolization therapy (Fig. 2B) over 3 sessions. In the first session $8 \mathrm{ml}$ of ethylene-vinyl alcohol copolymer (Onyx) was injected, resulting in embolization of approximately onequarter of the lesion. In the second session $4 \mathrm{ml}$ of Glubran was mixed in $30 \mathrm{ml}$ lipiodol, a radiopaque agent, and injected into the lesion. In the third session $17 \mathrm{ml}$ of Glubran mixed in $60 \mathrm{ml}$ lipiodol was injected (Fig. 2C and D). Five days following the second session the patient complained of nonspecific leg pains, which resolved approximately 5 days later without any specific intervention.

A decision was made to electively resect the lesion 2 weeks following the final embolization, but 5 days following the third embolization session, he developed suddenonset ataxia, tremor, and lethargy. The tremor was symmetrical and action induced, involving head, trunk, and limbs. There was no nystagmus or dysarthria. The patient's muscle strength and deep tendon reflexes were normal. He was irritable but his cognition was intact.

A CT scan of the brain showed subtle bilateral hyperdensities in the caudate nuclei, thalami, and cerebellar cortex (Fig. 3D). MRI of the brain was considered, but as there was concern regarding consequences of an electromagnetic heating effect of the intralesional Glubran, this was not undertaken. A chest radiograph showed diffuse interstitial changes consistent with interstitial pneumonitis (Fig. 3A). A CT scan of the thorax showed similar diffuse interstitial changes bilaterally with multiple areas of density in the perihilar and subcarinal regions (Fig. 3B).

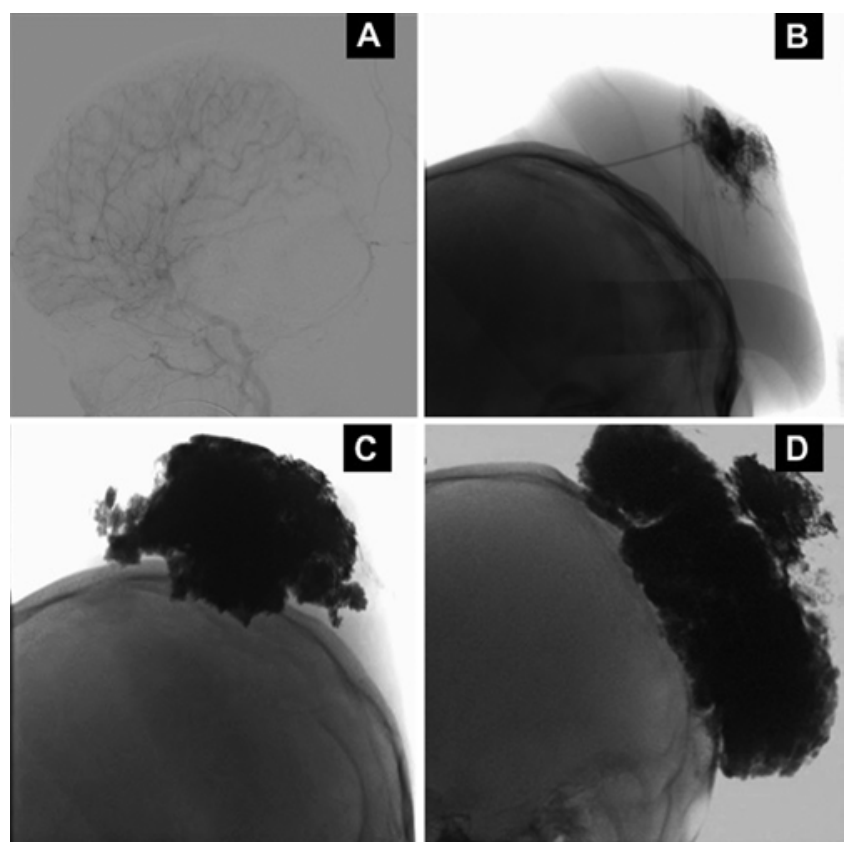

FIG. 2. Angiograms obtained before embolization (A) and immediately after the first (B), second (C), and third (D) embolization sessions. The second session was performed 5 days after the first, and the third was performed 7 days after the second.

Despite this finding, there were no respiratory signs or symptoms. A CT scan of the abdomen did not show any remarkable features (Fig. 3C).

Based on the clinical presentation and radiological findings, the possibility of toxicity secondary to Onyx or Glubran, or an immune-mediated reaction to either was considered, with an embolic phenomenon less likely due to the delay in onset of symptoms. Urgent resection of the hemangioma was scheduled.

Intraoperatively, the patient was positioned prone, and the hemangioma was carefully resected off the occiput and the underlying skull defect. No dural attachment was noted during resection and the skin was closed directly (Fig. 4).

On the 1st postoperative day, neurological symptoms worsened. Tremor was more pronounced but was only action induced. The patient was irritable and unable to stand or feed himself but had normal strength and preserved cognition.

Following a stat dose of intravenous dexamethasone (4 $\mathrm{mg}$ ), the symptoms decreased in severity. Dexamethasone was continued in addition to a 5-day course of $2 \mathrm{~g} / \mathrm{kg}$ intravenous immunoglobulin (IVIG). Clonazepam was prescribed for 72 hours for agitation and tremor. The patient's symptoms resolved over the following days and he was discharged home on a tapering dose of oral prednisolone. There was no blood eosinophilia, and histological examination of the resected hemangioma showed no eosinophilia or vasculitis. In retrospect, it would have been valuable to have had immunological investigations, but due to the sudden presentation, empirical treatment had to proceed, and fortunately the patient responded rapidly.

Neurological examination, a chest radiograph, and an 

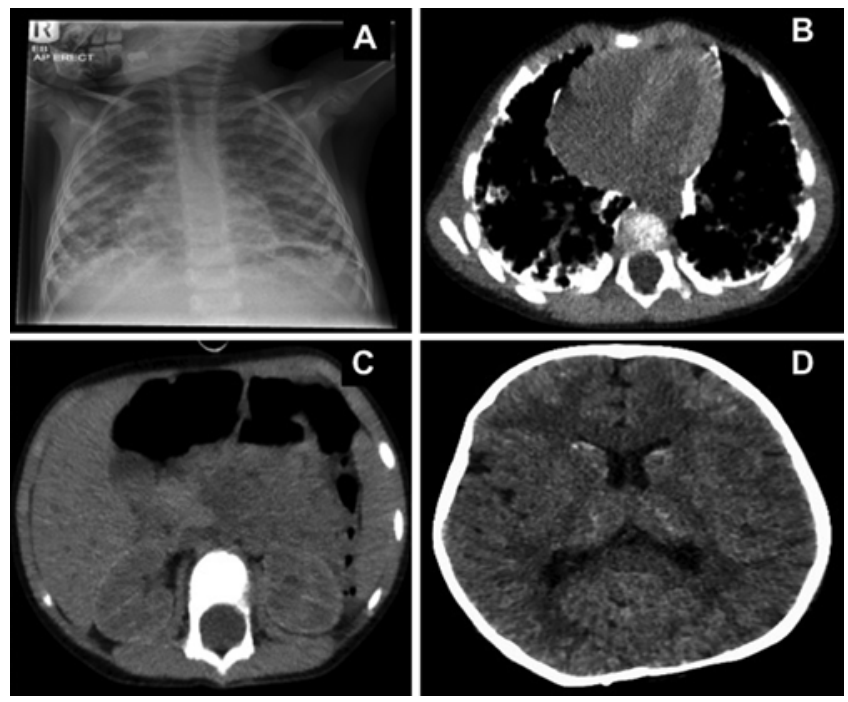

FIG. 3. Chest radiograph $(\mathbf{A})$ and axial CT images of the patient's thorax (B), abdomen (C), and brain (D) obtained 5 days after the third embolization session.

MRI study of the brain performed 6 months later showed no abnormality.

\section{Discussion}

This report describes an unexpected complication of Onyx and Glubran treatment of hemangioma. These materials are the most widely used liquid embolic agents in the world, but their adhesive properties and liquid nature require special precautions. ${ }^{1,7}$ Clinical manifestations in our patient may have been immune mediated. A prior report by Quinn et al. ${ }^{7}$ highlighted the potential for Glubran to produce an eosinophilic vasculitis in embolized intracranial arteriovenous malformations (AVMs). Additionally, other portions of nonembolized AVMs showed similar eosinophilic infiltrate, arguing that this is probably a nonfocal reaction. While none of these patients showed signs of systemic vasculitis, the authors concluded that long-term exposure to Glubran and subsequent development of an eosinophilic vasculitis in these patients may lead to fur-
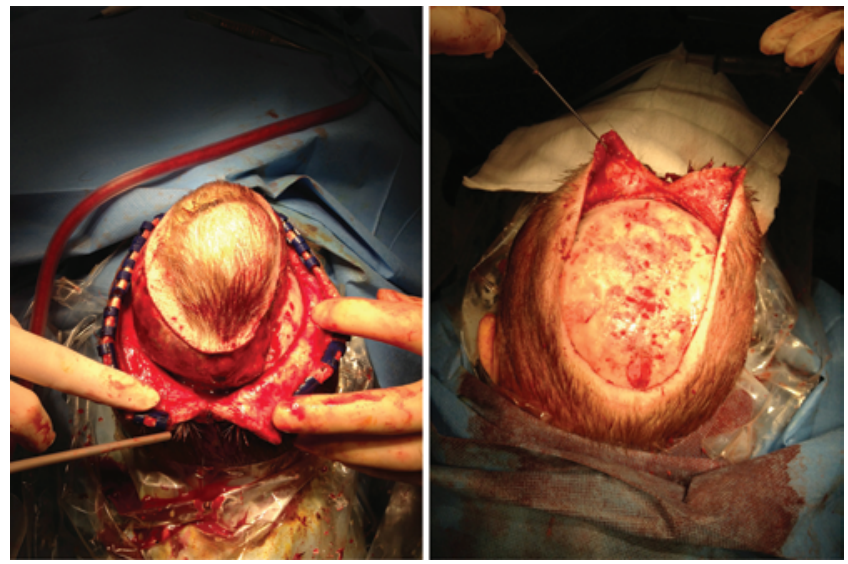

FIG. 4. Intraoperative photographs obtained during hemangioma resection (left) and closure (right). Figure is available in color online only. ther neurological sequelae or an increased risk of vessel rupture. Furthermore, a humoral immune response against Glubran may contribute to clinical symptoms.

We hypothesize that the neurological symptoms and radiological findings could be explained by an immunemediated process and therefore the patient was treated with steroids and IVIG. One week following discharge, symptoms had resolved and the child was neurologically normal. Although there were no blood or histological markers of an immune-mediated or allergic process, such a mechanism cannot be excluded.

While risks such as embolization to the venous system, intracranial hemorrhage, and pulmonary embolism have been reported in Glubran embolization of brain AVMs, ${ }^{1,4,5}$ an embolic phenomenon was not considered likely in this case due to the delay in onset of symptoms. Embolic events are more often instantaneous. Furthermore, there were no direct communicating or feeding vessels between the hemangioma and the systemic circulation. As the procedure was performed under continuous fluoroscopy, any potential migration of the Onyx or Glubran from the embolized area should have been visible. Relatively invisible communications between the hemangioma and systemic circulation may have facilitated the systemic toxicity or immune-mediated effect rather than the spread for emboli to multiple areas.

Despite the complications mentioned in the literature to date, none relate to the treatment of an extracranial lesion, as reported here. A definitive safe maximum dose has not yet been reported and it is largely driven by subsequent radiological appearance of the lesion as well as by the experience of the intervention radiologist. Murai et al. ${ }^{4}$ reported successful outcomes in 10 patients who underwent embolization for cerebellar hemangiomas with no reported side effects. The specific dose used is not stated. Effective therapy of Glubran treatment in laryngopharyngeal hemangioma has also been reported with a dose of $0.5-2 \mathrm{ml}^{2}$

In our case the dose used allowed successful and uncomplicated resection of what was initially a highly vascular lesion.

In summary, we present a significant and previously unreported side effect of Onyx and Glubran manifesting as a severe tremor, possibly immune mediated, following treatment of a giant congenital hemangioma. This complication should be borne in mind when using these embolic agents.

\section{References}

1. Ashour R, Aziz-Sultan MA, Soltanolkotabi M, Schoeneman SE, Alden TD, Hurley MC, et al: Safety and efficacy of Onyx embolization for pediatric cranial and spinal vascular lesions and tumors. Neurosurgery 71:773-784, 2012

2. Li W, Feng B, Wang J, Wu W, Liu L, Wang R, et al: [Observe the curative effect of n-butyl cyanoacrylate injection in treating laryngopharynx hemangioma.] Lin Chung Er Bi Yan Hou Tou Jing Wai Ke Za Zhi 22:1120-1122, 2008 (Chinese)

3. Marler JJ, Mulliken JB: Current management of hemangiomas and vascular malformations. Clin Plast Surg 32:99116, ix, 2005

4. Murai Y, Kominami S, Yoshida Y, Mizunari T, Adachi K, 
Koketsu K, et al: Preoperative liquid embolization of cerebeller hemangioblastomas using N-butyl cyanoacrylate. Neuroradiology 54:981-988, 2012

5. Niimi Y, Berenstein A, Setton A: Complications and their management during NBCA embolization of craniospinal lesions. Interv Neuroradiol 9 (Suppl 1):157-164, 2003

6. Picard A, Boscolo E, Khan ZA, Bartch TC, Mulliken JB, Vazquez MP, et al: IGF-2 and FLT-1/VEGF-R1 mRNA levels reveal distinctions and similarities between congenital and common infantile hemangioma. Pediatr Res 63:263-267, 2008

7. Quinn JC, Mittal N, Baisre A, Cho ES, Sharer LR, Gandhi C, et al: Vascular inflammation with eosinophils after the use of n-butyl cyanoacrylate liquid embolic system. J Neurointerv Surgu 3:21-24, 2011

\section{Disclosures}

The authors report no conflict of interest concerning the materi- als or methods used in this study or the findings specified in this paper.

\section{Author Contributions}

Conception and design: Kieran, Zakaria, Murray. Acquisition of data: Kieran, Laffan. Analysis and interpretation of data: Zakaria, Kaliaperumal, O'Rourke. Drafting the article: Kieran, Zakaria, O'Rourke. Critically revising the article: Kieran, Zakaria, Kaliaperumal, O'Hare, Laffan, Caird, King, Murray. Reviewed submitted version of manuscript: King. Approved the final version of the manuscript on behalf of all authors: Kieran. Administrative/ technical/material support: Laffan.

\section{Correspondence}

Ingrid Kieran, Department of Plastic Surgery, Temple Street Children's University Hospital, Temple St., Dublin, Ireland. email: ingridkieran@yahoo.com. 